\title{
Tangence
}

\section{La représentation de Montréal dans les images immobilisées du cinéma québécois}

\section{Michel Larouche}

Numéro 48, octobre 1995

Montréal et Vancouver : parcours urbains dans la littérature et le cinéma

URI : https://id.erudit.org/iderudit/025868ar

DOI : https://doi.org/10.7202/025868ar

Aller au sommaire du numéro

Éditeur(s)

Tangence

ISSN

0226-9554 (imprimé)

1710-0305 (numérique)

Découvrir la revue

Citer cet article

Larouche, M. (1995). La représentation de Montréal dans les images

immobilisées du cinéma québécois. Tangence, (48), 136-146.

https://doi.org/10.7202/025868ar d'utilisation que vous pouvez consulter en ligne.

https://apropos.erudit.org/fr/usagers/politique-dutilisation/ 


\section{La représentation de Montréal dans les images immobilisées du cinéma québécois Michel Larouche}

L'expression "images immobilisées" renvoie à la présence d'images fixes au cinéma, appelées souvent "photographies fixes" ou simplement "photographies". Si l'image fixe a toujours été présente dans l'histoire du cinéma, Metz avait déjà noté son importance parmi les traits narratifs particuliers du cinéma moderne ${ }^{1}$. Or depuis, ce procédé s'est considérablement accru. Il se généralise dans certaines cuvres expérimentales et apparaît de façon sporadique dans de nombreux autres films ${ }^{2}$.

Parmi les œuvres du cinéma québécois utilisant largement ce procédé, on note trois films d'importance: Albédo de Jacques Leduc et Renée Roy (1982), À corps perdu de Léa Pool (1988) et Les matins infidèles de Jean Beaudry et François Bouvier (1989). Dans les trois cas, l'utilisation de l'image fixe est liée à la représentation de Montréal et le mode discursif inclut la présence d'un photographe dans la diégèse des films.

\section{Des images de Montréal}

Dans Albédo, le personnage principal représente David Marvin, photographe et archiviste montréalais qui, le 6 juin 1975, choisissait de se suicider. Le film a pour sujet son drame, associé à la lente destruction de Griffintown, quartier dont l'archiviste avait retracé l'histoire. $\dot{A}$ corps perdu raconte le drame d'un reporter-photographe de profession qui, revenant d'une mission au

1 Christian Metz en parle en utilisant l'expression "photograpies fixes", dans Essais sur la signification au cinéma, tome 1, Paris, Klincksieck, 1971, p. 201.

2 À propos de cette problématique de l'image fixe au cinéma, voir nos précédentes publications: "Les enjeux de l'"effet photographie" au cinéma", Protêe, vol. 16, $\mathrm{n}^{\text {os }} 1-2$, hiver-printemps 1988, p. 134-138, et "Effet photo" et nouvelles images: vers un cinéma de l'affect ", Protée, vol. 19, n 3 , automne 1991 , p. 61-65. Le présent texte reprend et prolonge certaines des propositions avancées dans ces articles. 
Nicaragua, affronte le choc de la disparition du couple David et Sarah, avec lesquels il constituait un ménage à trois, et décide d'entreprendre un reportage sur Montréal. Les matins infidèles relate une tranche de vie de deux hommes dans la trentaine qui ont développé un projet commun à partir de l'idée de photoroman: alors que l'un, photographe, s'engage pendant un an à prendre un cliché chaque jour, à la même heure et toujours avec le même cadrage, d'un coin de rue d'un quartier de Montréal, l'autre, professeur et écrivain, rédige un roman à partir des photographies.

La présence d'images reliées à Montréal varie considérablement d'un film à l'autre: une diminution de leur importance peut être remarquée en fonction de leur adéquation à un réel concret.

Dans Albédo, elles relatent l'histoire de Griffintown: simple terrain vague dans l'île de Montréal à l'époque de la colonie, rapidement couvert de résidences au siècle dernier au moment de la construction du pont Victoria, il devient ce quartier situé entre la rue McGill à l'est, la rue Guy à l'ouest, la rue Notre-Dame au nord et le Canal Lachine au sud. Sa population sera formée en grande partie d'immigrés irlandais qui constituent une collectivité avec une vie communautaire bien à elle et que l'industrialisation va détruire. Nous assistons à une déconstruction de l'histoire dominante, qui ignore systématiquement les signes minoritaires du passé. Il y a renvoi significatif entre la surdité véritable de David Marvin et celle, trompeuse, de l'histoire. L'histoire d'un espace, d'une ville dans la ville que fut Griffintown, devient une contestation implicite d'un Montréal présent dont les traces historiques ont été évacuées. La voix off du personnage proclame: "Mon histoire commence avec Griffintown...". Le corps historique et géographique s'ancre sur le personnage individuel de Marvin et met en valeur une dimension socio-politique.

Dans $\bar{A}$ corps perdu, les images fixes présentent des rues désertes avec gratte-ciel et autoroutes en béton, et une iconographie montrant des quartiers en démolition: terrains vagues, murs avec graffiti, bâtiments abandonnés aux fenêtres brisées, entrepôts gagnés par la rouille, souterrains, etc. Le cinéphile peut y reconnaître le vieil entrepôt coin Amherst et Saint-Antoine, maintes fois présenté dans le cinéma québécois.

Les matins infideles propose un point de vue spécifique: un snack-bar, un arrêt d'autobus et un jeune érable étouffé par le 
138

béton. La banalité de l'atmosphère quotidienne typique d'un quartier populaire de Montréal, le Plateau Mont-Royal, est ici mise en valeur.

Les images fixes de ces trois films ne proposent pas un Montréal homogène mais un Montréal de la marge. Les perspectives diffèrent considérablement d'un film à l'autre et se distinguent par leur singularité. En même temps, ces images sont très près de la représentation de Montréal que le cinéma québécois a toujours véhiculée:

Toutes ces vues fragmentaires, toutes ces "petites patries" ne trahissent-elles pas la nostalgie du village imaginaire, ne suggèrent-elles pas une certaine incapacité d'enserrer la ville à bras le corps, ne démontrent-elles pas le désir de l'attache parcellaire? Quant aux intérieurs, si nombreux, peut-on en définitive les distinguer de ceux de Trois-Rivières ou de Sherbrooke? D'autre part, le centre-ville de Montréal n'acquiert souvent au cinéma que le statut de plan de raccord; c'est un lieu de passage et de mouvement; on s'y arrête peu, on y vit moins encore. On en fait le trait d'union entre deux aventures, mais un trait d'union qui, paradoxalement, indique clairement sa nature urbaine car, si la route évoque le voyage et le road movie, la rue appelle la circulation, autrement dit la mobilité, le déplacement, la fluidité $^{3}$

Mais les trois films qui nous intéressent partagent deux caractéristiques: images fixes et acte photographique, qui constituent en fait des éléments d'une stratégie discursive commune qui influe sur la représentation de Montréal et laisse percer, au-delà des disparités, une figure unique et nouvelle d'une vaste instance discursive, confirmant l'intérêt d'aborder les trois films à la façon d'un texte plurifilmique.

\section{Un mode discursif}

Le cinéma classique propose au spectateur un récit et un espace imaginaire: se construit alors un "centre " à partir duquel s'établit la cohérence de la chaîne narrative. Ce dispositif relie étroitement analogie photographique et indices de réalité. Il y a

3 Pierre Véronneau, "En vedette au Montréaloscope... ", Pierre Véronneau (dir.), Montréal ville de cinéma, Montréal, Cinémathèque québécoise/Musée du cinéma, 1992, p. 3. 
aussi des règles de correspondance entre les divers points de vue. Jacques Aumont spécifie par rapport au cinéma l'éventail des significations de la locution "point de vue " ${ }^{4}: 1$. L'emplacement de la caméra relativement à l'objet regardé; 2 . La vue ellemême, c'est-à-dire le cadre avec effet de surface et illusion de profondeur; 3. Le point de vue narratif (représentation d'un regard, celui de l'auteur ou d'un personnage); 4 . Le point de vue prédicatif, sorte de surdétermination par le travail de la représentation. Les règles d'harmonisation des points de vue opèrent en fonction de la mise en valeur du point de vue narratif, l'illusion de profondeur l'emportant sur l'effet de surface et le travail sur le signifiant. Ainsi se constitue le "bon espace".

Par rapport à ce régime, l'image fixe produit un "brouillage" spatio-temporel par une modification du temps - l'image isolée est un punctum temporis que l'on a immobilisé (Metz) - et une parcellisation de l'espace. Comme le note Jean Mitry: "Ce n'est que lorsque la même photographie est invariablement répétée que se produit la sensation d'effondrement et d'applatissement. " 5 L'ordre de correspondance des points de vue est donc renversé, le point de vue narratif se trouvant en quelque sorte suspendu au profit des autres points de vue: l'emplacement depuis lequel on regarde, l'occupation plastique de la surface du cadre et le travail de représentation.

Il va de soi que lorsqu'on fait jouer de manière "photographique. le dispositif d'énonciation même du cinéma, lorsqu'on touche aux composantes du langage filmique lui-même, on introduit des altérations fondamentales. On est parfois à la limite du cinéma dit expérimental (expérience d'image, à nouveau, expérience-mise à l'épreuve du cinéma par la photo). ${ }^{6}$

L'image fixe nie en quelque sorte la narrativité en donnant l'importance à la présence concrète, en produisant une surcharge figurative au détriment de la figuration indirecte inscrite dans le récit, tout en pointant le processus narratif lui-même. Johan van der Keuken disait à ce propos :

4 Jacques Aumont, "Le point de vue", "Énonciation et cinéma ", Communications, $\mathrm{n}^{\circ} 38$, Paris, Seuil, 1983, p. 14-15.

5 Jean Mitry, Esthétique et psycbologie du cinéma, tome 2: Les structures, Paris, Éd. Universitaires, 1963 , p. $169-170$.

6 Philippe Dubois, "Présentation", "Films de photos", Revue belge du cinêma, $\mathrm{n}^{\mathrm{O}} 4$, été 1983, p. 2. 
Film-de-photographie: qu'y a-t-il de plus palpitant que la presque immobilité, que la réalité très visiblement découpée par un cadre qui est presque définitif, mais qui éclate au dernier moment, en haut, en bas, sur les côtés, vers d'autres visions? La photographie ne peut pas faire cela. Seul un moyen d'expression animé peut montrer l'immobilité et le retour vers le mouvement. ${ }^{7}$

Si la photographie comme telle tend à un certain décalque perceptif, l'image fixe au cinéma se présente comme une situation fortement codée, laissant transparaître l'ostentation et la volonté de faire signifier par-delà sa représentation, forçant à reconsidérer ce qui se faufile, glisse dans le discours filmique. L'image fixe constitue un aveu de montage dont l'effet se rabat sur l'ensemble du film. "Une telle immobilisation, voire paralysie fascinante de l'objet ou de la personne, fait naitre l'agitation la plus intense " 8 , dit Nicole Gingras.

Ces images fixes illustrant Montréal, dans les films qui font l'objet de notre étude, font en sorte que sa représentation s'en voit d'autant plus marquée. Trois volets conducteurs se développent, se côtoient, s'entrecroisent dans Albédo: l'histoire d'un individu, l'histoire d'un quartier disparu de Montréal et l'histoire d'un couple. Il y a dégradation dans les trois cas. Les images de Montréal, autorisant le passage d'un volet à l'autre, permettent le déploiement de l'architecture du film. Dans $\bar{A}$ corps perdu, les images de Montréal reflètent les états d'âme du photographe Kurwenal, agissant comme point de convergence des émotions et des angoisses qui caractérisent sa cacophonie intérieure. Les clichés photographiques des Matins infidèles nous permettent de pénétrer dans l'univers des personnages: si la simple alternance des photographies semble au départ anodine, elle révèle, progressivement, la complexité des deux êtres.

Les images de Montréal apparaissent donc liées à un dispositif particulier, sorte de mise en scène de la fixité au cinéma, qui obligent le spectateur non plus à s'abolir dans le processus de "fictionnalisation ", mais à regarder. L'aspect contemplatif présent dans tout film n'est plus contredit par la mécanique narrative, il est ici

7 Johan Van der Keuken, Voyage à travers les tours d'une spirale, Montréal, Cinémathèque québécoise, 1986, p. 55.

8 Nicole Gingras, Les images immobilisées. Procéder par impressions, Montréal, Guernica, 1991, p. 48. 
mis de l'avant. Nous assistons alors à une "dé-hiérarchisation" et à une "dé-linéarisation" d'un dispositif en fonction de la mise en valeur de ce que Barthes appelle le punctum en photographie: "Le punctum est alors une sorte de hors-champ subtil, comme si l'image lançait le désir au-delà de ce qu'elle donne à voir [...]."? Les images immobilisées de Montréal ne sont plus des points de vue à l'intérieur d'un ensemble d'images mouvantes, mais une véritable clef de voûte. Leur singularité leur confère un statut privilégié, l'"histoire [devenant] en quelque sorte paramétrique au signifiant dont elle n'est plus que le champ de déplacement, la négativité constitutive, ou encore: la compagne de route " ${ }^{10}$, pour reprendre les propos de Barthes dans "Le troisième sens".

Le Montréal tel qu'illustré par les images fixes devient ainsi une figure qui polarise la perception du spectateur et son mode d'adhésion au film. Afin de cerner ce rapport spectateur-film via Montréal, il faut cependant voir, au-delà de l'identification des images et de leur importance dans le processus des oeuvres, comment se dessine cette figure. Il s'agit d'un Montréal disparu dans Albédo, le quartier Griffintown du Centre-sud n'ayant laissé aucune trace en dehors des photographies et documents d'archives. Un Montréal impersonnel dans $\ddot{A}$ corps perdu. Parlant de la transposition du roman d'Yves Navarre, la cinéaste précise en effet: "Toutes les villes finalement se ressemblent. Il n'a pas été difficile de replacer l'action à Montréal. "11 Un Montréal qui semble particularisé dans Les matins infideles (un coin de rue), mais qui ne fait pas partie, dans la réalité profilmique, du quartier désigné: la photographie a été prise coin Saint-Zotique et SaintVallier et non coin "Duluth et Saint-Urbain", titre de travail du film. La répétition, inhérente au concept, traduit d'ailleurs la dimension plutôt intemporelle du secteur.

La figure de Montréal fait ressurgir traces, marques, cicatrices, vagues de temps oublié tout en conjurant la disparition inévitable, déstabilisant de la sorte la perception de la ville traduite par les images en mouvement.

9 Roland Barthes, La chambre claire. Note sur la photographie, Paris, Cahiers du cinéma/Gallimard/Seuil, 1980, p. 93.

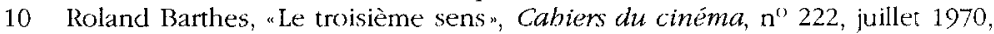
p. 18 .

11 Propos cités par Luc Perreault, "Dans la fumée du Kilomètre Heure... Léa Pool tourne Kurwenal ", La Presse, 21 novembre 1987, p. E 17. 
142

Les affects des spectateurs se voient canalisés par des enjeux qui sont propres à la logique photographique. Philippe Dubois les résume de la façon suivante:

[...] figement, raidissement, pétrification (effet-Méduse de la photo), érection et castration, coupure, prélèvement, décollement, fascination et répulsion, pulsion de vie et désir de mort, puissance mortifère du regard, sexualisation de la prise, etc. ${ }^{12}$

Ces enjeux, en fait, consistent en des dispositifs analogiques dont la virtualité extensive et la force d'expansion métonymique est très grande. Montréal apparaît donc comme une figure labyrinthique.

\section{Une matrice virtuelle}

Mais qu'elle apparaisse totalement ou partiellement dans le film, l'image fixe entraîne une appréhension conceptuelle de l'espace et du temps qui tient compte de l'outillage, ce qui caractériserait la pensée postmoderne. Daniel Charles précise à ce sujet dans "Présence et post-modernitén:

[...] on assiste (et participe) au rassemblement-éclair, en une seule gerbe, des trois dimensions du temps, avenir-passé-présent. Le temps post-moderne est celui de la Gleichzeitigkeit, de l'équitemporalité" selon Heidegger - mais celle-ci est médiatisée par le Gestell, par la technique comme telle. ${ }^{13}$

Le spectateur n'est plus investi de l'extérieur par un réseau spatiotemporel, il collabore à l'émergence d'un réseau où le medium technique a déjà opéré. Il performe. C'est l'expérience du spectateur qui produit les conditions de perception. L'ounre devient le lieu de coexistence de modalités perceptuelles diverses en puissance pour et par le spectateur. Si performer, c'est transformer, c'est à des séries de transformations que ces films invitent le spectateur: transformations que la technique impose à l'expérience perceptive, transformations que les spectateurs font subir à l'expérience médiatisée, transcodages donc.

Le personnage principal, dans les trois films de notre corpus, étant photographe, cette "agitation" du spectateur est multipliée.

12 Philippe Dubois, op. cit.

13 Daniel Charles, "Présence et post-modernitén, Hos Cadre, n" 3, printemps 1985. p. 160 . 
L'effet de surface créant fascination, dérive sémantique et polysémie se répercute sur le processus de "fictionnalisation" favorisant l'identification et instaurant une sorte de "surrégime "filmique. Hyperactif, sans repos, toujours sur le qui-vive, en quête de scènes à fixer, tout dans l'instantanéité de la prise de vue fait impression sur le photographe et se répercute sur l'activité spectatorielle. Un déplacement s'opère de la photographie à la création en général.

L'albédo", terme utilisé en astronomie, est, nous dit-on dans le film, le rapport entre la lumière incidente et la lumière réfléchie. Ainsi, Vénus et la Terre ont un albédo respectif ; la neige aussi. Par extension et transposition, on peut dire qu'une photographie peut avoir un albédo ou être l'albédo de quelque chose. Le film devient une réflexion sur l'apparence et la réalité des choses et des gens, sur ce qui est réfléchi par l'un et l'autre. Tout est une question d'incidence, d'angle entre l'observateur et l'observé, de mode entre la représentation et le représenté. Montréal dans Albédo, c'est l'albédo de Montréal: une question de point de vue.

Dans $\dot{A}$ corps perdu, Kurwenal entreprend un reportage sur sa ville lorsqu'il est confronté à lui-même, peut-être pour la première fois. Les images en noir et blanc de la ville se voient associées aux souvenirs qui le hantent. Il découvre un Montréal ignoré comme le révélateur qui dévoile peu à peu l'image latente. "[...] Il y a effectivement un questionnement sur la lumière - dit Léa Pool -, l'espace et en fait, sur la création visuelle. Le processus créatif porte mes histoires et dévoile l'intériorité de mes personnages. Tout cela est étroitement lié "14.

Le film Les matins infidèles entretient aussi des relations privilégiées entre le réel et l'imaginaire, dimension qui ressort lorsque le photographe commence à tricher avec la réalité et lance son ami romancier sur de nouvelles pistes. Il recule d'abord l'heure, puis enlève ensuite l'horloge à la vitrine du snack-bar, évacuant totalement le temps, et il projette enfin ses fantasmes amoureux dans la photographie. La banalité du coin de rue, modifiée par les mises en scène de l'un, sera alors transformée par les fantasmes de l'autre. La photographie n'est plus "ce qui a eu lieu", mais "ce qui est".

14 Propos cités par Claudette Thériault, a ̀̀ corps perdu, adaptation cinématographique du livre de Yves Navarre, Kurwenal "L'Atiron, 18 janvier 1989. 
144

Montréal devient une figure génératrice, une sorte d'opérateur de vertige, d'espace du possible " 15 pour reprendre l'expression de Pierre Nepveu et Gilles Marcotte dans leur introduction à Montréal imaginaire, une matrice virtuelle.

Le fait que les images de Montréal n'aient pas une égale importance dans leur adéquation à un réel concret, dans Albédo, $\dot{A}$ corps perdu et Les matins infidèles ne devient plus un élément véritablement significatif, le point de vue sur la ville se situant ailleurs. Plaque tournante permettant à la fois une relation ternaire aux films qui favorise l'identification à travers le personnage principal du photographe, et une relation duelle reliée à un effet de surface, l'"effet image" l'emportant sur l'effet fiction, la ville surgit "en creux", tient lieu de négatif. Et par effet d'impression, elle laisse surgir une ville jumelle, le Montréal des images mouvantes. Un Montréal de parcours, de choix et d'interprétations.

15 Pierre Nepveu et Gilles Marcotte (dir.), Montréal imaginaire, Ville et littérature, Montréal, Fides, 1992, p. 8. 


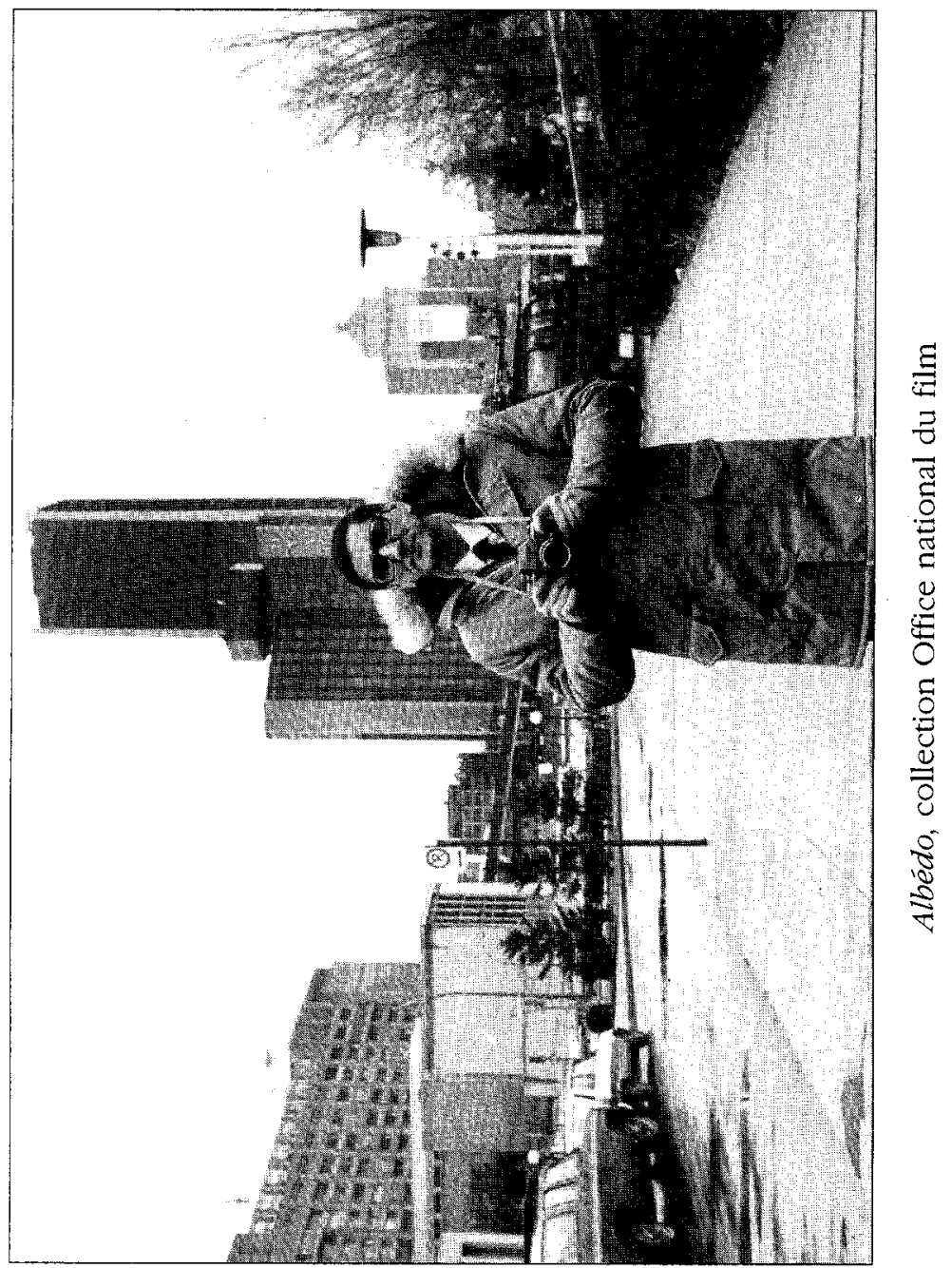




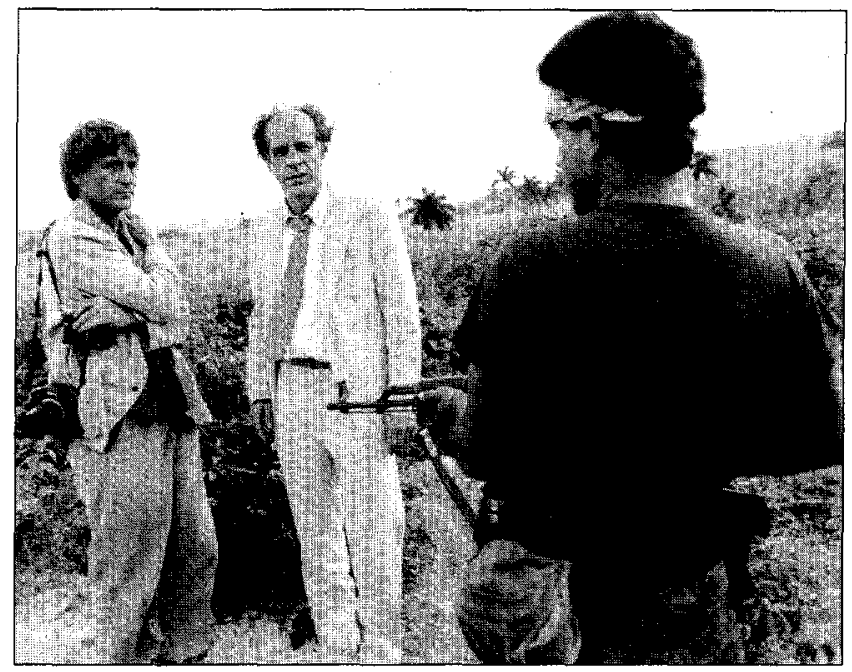

À corps perdu, collection Cinémathèque québécoise

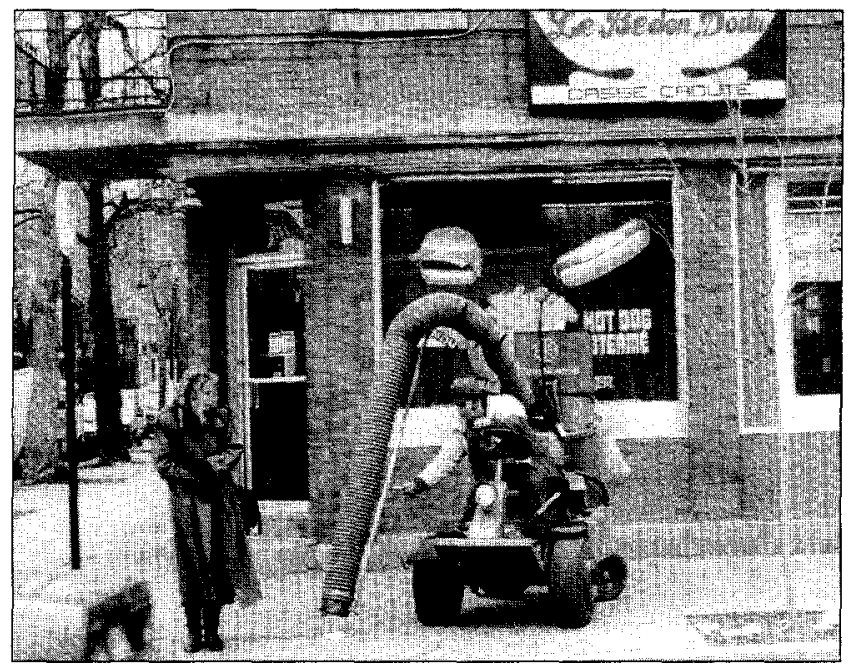

Les matins infidèles, collection Cinémathèque québécoise 